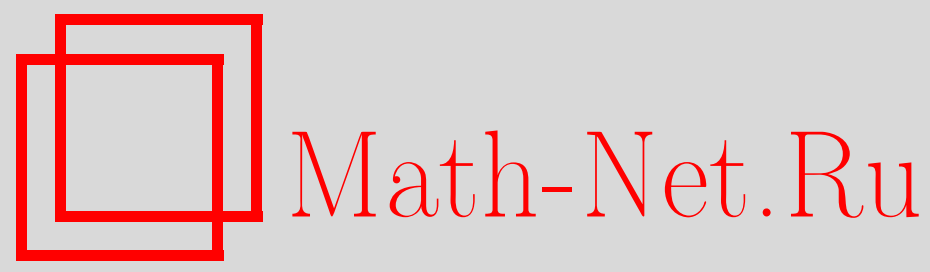

И. А. Солдатенков, Об использовании преобразования Фурье для решения сингулярных интегральных уравнений, Матем. заметки, 2003, том 74, выпуск 5, 739-744

DOI: https://doi.org/10.4213/mzm306

Использование Общероссийского математического портала Math-Net.Ru подразумевает, что вы прочитали и согласны с пользовательским соглашением http://www.mathnet.ru/rus/agreement

Параметры загрузки:

IP : 54.164 .48 .24

26 апреля 2023 г., 14:49:56

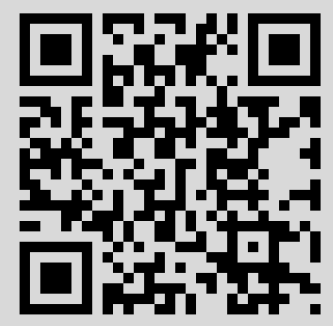




\title{
ОБ ИСПОЛЬЗОВАНИИ ПРЕОБРАЗОВАНИЯ ФУРЬЕ ДЛЯ РЕШЕНИЯ СИНГУЛЯРНЫХ ИНТЕГРАЛЬНЫХ УРАВНЕНИЙ
}

\section{И. А. Солдатенков}

\begin{abstract}
Предлагается метод решения системы сингулярных интегральных уравнений с постоянными коэффициентами. В основе метода лежит процедура сведения сингулярных уравнений к уравнениям с непрерывным разностным ядром, решение которых строится с помощью классического преобразования Фурье в классе абсолютно интегрируемых функций. Получены явные выражения для решения рассматриваемых сингулярных интегральных уравнений.
\end{abstract}

Библиограффия: 7 названий.

1. Введение. Излагаемьй ниже материал касается построения в замкнутом виде решения системы линейных сингулярных уравнений с постоянными коэффициентами

$$
A q(x)+B \int_{-a}^{a} \frac{q(\xi) d \xi}{\xi-x}=f(x), \quad x \in(-a, a)
$$

где $A, B$ - постоянные матрицы $N \times N, N=1,2, \ldots, f(x)$ и $q(x)$ - заданная и искомая вектор-функции размерности $N, x$ - действительная переменная.

Классический подход к решению систем (1) основывается на их сведении к задаче линейного сопряжения для нескольких функций комплексного переменного [1], [2]. При таком подходе удается построить решения более общих, чем (1), систем сингулярных интегральных уравнений, допускающих, в частности, зависимость матрищ $A, B$ от $x$ и более сложные контуры интегрирования. Однако соответствующие алгоритмы построения решения включают в себя достаточно трудоемкие процедуры нахождения канонических решений задачи сопряжения, в результате чего построение в замкнутом виде решения уравнения (1) на основе классического подхода представляется затруднительным.

2. Основные соотношения. Запись $\varphi(x) \in H^{*}[-a, a]$ означает, что

$$
\varphi(x)=\varphi^{*}(x)(a+x)^{-\varepsilon_{1}}(a-x)^{-\varepsilon_{2}}, \quad \varphi^{*}(x) \in H[-a, a], \quad \varepsilon_{k}<1,
$$

Работа выполнена при финансовой поддержке Российского фонда фундаментальных исследований, грант № 01-01-00034, и Международной ассоциации по содействию сотрудничеству с учеными из независимых государств бьвшего Советского Союза (INTAS), грант № 99-0671. 
где $H[-a, a]$ - класс Гёльдера [1]. Предполагается, если другое не оговорено, что компоненты вектор-функций $f(x), q(x)$ содержатся в $H^{*}[-a, a]$. Не ограничивая общности, компоненты $f, q, A, B$ будем считать действительньми, причем $\operatorname{det} A \neq 0$.

Преобразуем систему (1). Умножим ее на $(x-t)^{-1}$ при некотором $t \in(-a, a)$ и проинтегрируем по $x \in[-a, a]$. Затем, полагая $\operatorname{det} A \neq 0$, умножим полученное равенство на $B A^{-1}$ и, пользуясь опять (1), заменим в нем интеграл $B \int_{-a}^{a} \frac{q(x) d x}{x-t}$ на $f(t)-A q(t)$. Тогда можно прийти к уравнению

$$
A q(x)+n \int_{-a}^{a} \frac{d t}{t-x} \int_{-a}^{a} \frac{q(\xi) d \xi}{\xi-t}=d(x),
$$

в котором $n=-B A^{-1} B$,

$$
d(x)=f(x)-B A^{-1} \int_{-a}^{a} \frac{f(\xi) d \xi}{\xi-x} \in H^{*}[-a, a]
$$

УТВЕРЖДЕНИЕ 1. Множсество решений уравнения (3) содержит все решения исходного уравнения (1).

В дальнейшем также будет использоваться следующая эквивалентная (3) запись, которая получается, если в (3) произвести перестановку порядка интегрирования на основе формулы Пуанкаре-Бертрана [1]:

$$
m q(x)+n \int_{-a}^{a} k(\xi, x) q(\xi) d \xi=d(x)
$$

где $m=A-\pi^{2} n, k(\xi, x)=\frac{L(\xi)-L(x)}{\xi-x}, L(x)=\ln \left|\frac{a+x}{a-x}\right|$.

3. Решение уравнения (3) класса $H_{1 / 2}^{*}$. Запись $\varphi(x) \in H_{1 / 2}^{*}[-a, a]$ означает, что функция $\varphi(x)$ представима в виде $(2)$ при $\varepsilon_{k}<1 / 2$. Будем здесь полагать, что $f(x)$ и $q(x)$ принадлежат $H_{1 / 2}^{*}[-a, a]$, при этом функция $d(x)$ вида $(4)$ также будет содержаться в $H_{1 / 2}^{*}[-a, a][1]:$

$$
\{f(x), q(x), d(x)\} \in H_{1 / 2}^{*}[-a, a] .
$$

Введем новую переменную $s$ и соответствующие ей функции

$$
2 s=L(x), \quad x=a \text { th } s, \quad Q(s)=q(a \text { th } s), \quad D(s)=d(a \text { th } s) .
$$

Тогда уравнению (5) можно придать вид

$$
m \Phi(s)+2 n \int_{-\infty}^{\infty} \frac{(s-\eta)}{\operatorname{sh}(s-\eta)} \Phi(\eta) d \eta=R(s)
$$

где $\Phi(s)=a Q(s) / \operatorname{ch} s, R(s)=a D(s) / \operatorname{ch} s$, причем в силу (6)

$$
\{\Phi(s), R(s)\} \in H(-\infty, \infty) \cap L(-\infty, \infty) .
$$


Здесь $H(-\infty, \infty)$ обозначает класс функций, удовлетворяющих условию Гёльдера в некоторой окрестности любой точки действительной оси.

Далее будет использоваться классическое преобразование Фурье [3]:

$$
\tilde{f}(\lambda)=\frac{1}{\sqrt{2 \pi}} \int_{-\infty}^{\infty} f(s) e^{i \lambda s} d \lambda, \quad f(s)=\frac{1}{\sqrt{2 \pi}} \mathrm{v} \cdot \mathrm{p} \cdot \int_{-\infty}^{\infty} \tilde{f}(\lambda) e^{-i \lambda s} d \lambda
$$

и следующие соотношения для свертки $(f * g)(s)$ :

$$
(f * g)(s) \in C(-\infty, \infty) \cap L(-\infty, \infty), \quad \widetilde{(f * g)}(\lambda)=\tilde{f}(\lambda) \tilde{g}(\lambda)
$$

при $\{f(s), g(s)\} \in C(-\infty, \infty) \cap L(-\infty, \infty),|f(s)| \leqslant$ const;

$$
(f * g)(s)=\frac{1}{\sqrt{2 \pi}} \mathrm{v} \cdot \mathrm{p} \cdot \int_{-\infty}^{\infty} \tilde{f}(\lambda) \tilde{g}(\lambda) e^{-i \lambda s} d \lambda
$$

при $\{f(s), g(s), \tilde{f}(\lambda)\} \in L(-\infty, \infty), f(s) \in H(-\infty, \infty), g(s) \in C(-\infty, \infty)$.

Воздействуем на обе части уравнения (8) преобразованием Фурье, при этом воспользуемся вторым соотношением (10), учитьвая (9). В результате, обозначая

$$
K_{a}(\lambda)=m \zeta(\lambda)+\pi^{2} n, \quad \zeta(\lambda)=\operatorname{ch}^{2} \frac{\pi}{2} \lambda
$$

получим равенство $K_{a}(\lambda) \widetilde{\Phi}(\lambda)=\zeta(\lambda) \widetilde{R}(\lambda)$, в котором, учитывая $(9), \widetilde{R}(\lambda) \in C(-\infty, \infty)$, $\lim _{\lambda \rightarrow \pm \infty} \widetilde{R}(\lambda)=0,|\widetilde{R}(\lambda)| \leqslant$ const [3]. Полученное равенство представляет собой алгебраическое уравнение для $\Phi$ урье-образа $\widetilde{\Phi}(\lambda)$ неизвестной функции $\Phi(s)$, условие разрешимости которого имеет вид [4]: $\operatorname{det} K_{a}(\lambda) \neq 0, \lambda \in(-\infty, \infty)$. В этом случае существует матрища $\tilde{h}_{a}(\lambda)=K_{a}^{-1}(\lambda)$ и

$$
\widetilde{\Phi}(\lambda)=\zeta(\lambda) \tilde{h}_{a}(\lambda) \widetilde{R}(\lambda) .
$$

Представляя $\tilde{h}_{a}(\lambda)=M(\lambda) / \operatorname{det} K_{a}(\lambda)$, где $M(\lambda)$ - матрица $N \times N$ алгебраических дополнений элементов матрицы $K_{a}(\lambda)$, и полагая $\operatorname{det} m \neq 0$, можно установить следующие свойства $\tilde{h}_{a}(\lambda)$ :

$$
\begin{gathered}
\left(1+|\lambda|^{l}\right) \tilde{h}_{a}^{(k)}(\lambda) \in C(-\infty, \infty) \cap L(-\infty, \infty), \\
\lim _{\lambda \rightarrow \pm \infty} \lambda^{l} \tilde{h}_{a}^{(k)}(\lambda)=0, \quad k, l=0,1, \ldots,
\end{gathered}
$$

из которых вытекает существование Фурье-прообраза $h_{a}(s)$ матричной функции $\tilde{h}_{a}(\lambda)$ такого, что [3]

$$
\lim _{s \rightarrow \pm \infty} s^{k} h_{a}^{(l)}(s)=0, \quad h_{a}^{(l)}(s) \in C(-\infty, \infty) \cap L(-\infty, \infty), \quad k, l=0,1,2, \ldots
$$

Представим (12) в виде равенства $\widetilde{\Phi}(\lambda)=m^{-1} \widetilde{R}(\lambda)-\tilde{h}_{a}(\lambda) \pi^{2} n m^{-1} \widetilde{R}(\lambda)$ и воздейст-

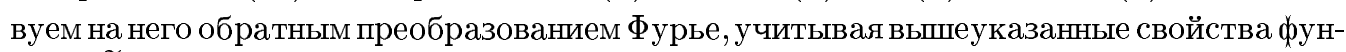
кции $\widetilde{R}(\lambda)$, а также $(9),(11),(13)$ и (14). В результате, если вернуться к функциям 
$Q(s)=a^{-1} \Phi(s) \operatorname{ch} s, D(s)=a^{-1} R(s) \operatorname{ch} s$, можно получить следующее выражение для $Q(s)$ :

$$
Q(s)=m^{-1} D(s)-\frac{1}{\sqrt{2 \pi}} \operatorname{ch} s \int_{-\infty}^{\infty} h_{a}(s-\eta) \pi^{2} n m^{-1} D(\eta) \frac{d \eta}{\operatorname{ch} \eta}
$$

Выражение (15) удовлетворяет сделанному допущению (6), если усилить первое свойство (14) и потребовать экспоненциального затухания на бесконечности известной матричной функции $h_{a}(s)$ и ее производной:

$$
h_{a}^{(l)}(s)=\mathscr{O}\left(\operatorname{ch}^{-\beta} s\right), \quad \beta>0, \quad l=0,1 .
$$

Ниже будет рассмотрен вопрос о возможности использования выражения (15) в качестве решения исходного уравнения (1).

4. Решение уравнения (3) класса $H^{*}$. Далее вместо (6) положим

$$
\{f(x), q(x), d(x)\} \in H^{*}[-a, a] .
$$

Для построения решения уравнения (3) из класса $H^{*}[-a, a]$ выполним тождественное преобразование этого уравнения. А именно, обратим дважды повторньй интеграл в (3) с помощью известной формулы [1], [5]:

$$
\frac{1}{\pi} \int_{-a}^{a} \frac{\varphi(\xi) d \xi}{\xi-x}=\psi(x) \Longleftrightarrow \varphi(x)=\frac{1}{\pi \sqrt{a^{2}-x^{2}}}\left[\int_{-a}^{a} \varphi(x) d x-\int_{-a}^{a} \frac{\sqrt{a^{2}-\xi^{2}} \psi(\xi)}{\xi-x} d \xi\right] .
$$

В результате можно получить

$$
\begin{aligned}
m \sqrt{a^{2}-x^{2}} q(x)-\frac{A}{\pi^{2}} \int_{-a}^{a} \sqrt{a^{2}-\xi^{2}} q(\xi) k(\xi, x) d \xi \\
=\sqrt{a^{2}-x^{2}} d(x)-\frac{1}{\pi^{2}} \int_{-a}^{a} \sqrt{a^{2}-\xi^{2}} d(\xi) k(\xi, x) d \xi \\
\quad-\left(\pi n P^{0}+\frac{1}{\pi} n P^{1} L(x)\right)
\end{aligned}
$$

где

$$
P^{0} \equiv \int_{-a}^{a} q(x) d x, \quad P^{1} \equiv \int_{-a}^{a} d x \int_{-a}^{a} \frac{q(\xi) d \xi}{\xi-x}=\int_{-a}^{a} q(x) L(x) d x
$$

По аналогии с предыдушим разделом можно ввести переменную $s$ и соответствующие ей функции $Q(s), D(s)($ см. (7)), а также функции

$$
\Psi(s)=\frac{a^{2} Q(s)}{\operatorname{ch}^{2} s}, \quad W(s)=\frac{a^{2} D(s)}{\operatorname{ch}^{2} s} .
$$


Используя их, можно представить уравнение (18) в аналогичном (8) виде, после чего, воздействуя на него преобразованием Фурье, прийти, как и в предыдущем разделе, к следуюшему выражению для $Q(s)$ :

$$
\begin{aligned}
Q(s)= & m^{-1} D(s)+\frac{1}{\sqrt{2 \pi}} \operatorname{ch}^{2} s \int_{-\infty}^{\infty} h_{b}(s-\eta)\left(A m^{-1}-E\right) D(\eta) \frac{d \eta}{\operatorname{ch}^{2} \eta} \\
& -\frac{1}{a^{2}} \operatorname{ch}^{2} s\left[\Gamma_{0}(s) P^{0}+\Gamma_{1}(s) P^{1}\right]
\end{aligned}
$$

где $E$ - единичная матрица, $h_{b}(s)$ - Фурье-прообраз матричной функции $\tilde{h}_{b}(\lambda)=$ $K_{b}^{-1}(\lambda), K_{b}(\lambda)=m \zeta(\lambda)-A, \Gamma_{0}(s), \Gamma_{1}(s)$ - Фурье-прообразы матричных функций $\widetilde{\Gamma}_{0}(\lambda)=\sqrt{\pi / 2} \pi a \operatorname{ch}(\pi \lambda / 2) \tilde{h}_{b}(\lambda) n, \widetilde{\Gamma}_{1}(\lambda)=i \sqrt{\pi / 2} a \operatorname{sh}(\pi \lambda / 2) \tilde{h}_{b}(\lambda) n$. Существование функции $\tilde{h}_{b}(\lambda)$ обеспечивается условием [4] $\operatorname{det} K_{b}(\lambda) \neq 0, \lambda \in(-\infty, \infty)$, при этом выражение (18) удовлетворяет сделанному допущению (17), если

$$
h_{b}^{(l)}(s)=\mathscr{O}\left(\operatorname{ch}^{-\beta} s\right), \quad \Gamma_{0,1}^{(l)}(s)=\mathscr{O}\left(\operatorname{ch}^{-\varepsilon} s\right), \quad \beta>0, \quad \varepsilon>0, \quad l=0,1 .
$$

5. Решение исходного уравнения (1). Вьше был осушествлен переход от уравнения (1) к уравнению (3), решение $q(x) \in H_{1 / 2}^{*}[-a, a]$ которого имеет вид (15) при условиях $\operatorname{det} K_{a}(\lambda) \neq 0, \lambda \in(-\infty, \infty),(6)$ и (16), тогда как решение $q(x) \in H^{*}[-a, a]$ определяется формулой (20) при условиях $\operatorname{det} K_{b}(\lambda) \neq 0, \lambda \in(-\infty, \infty),(17)$ и $(21)$, причем в обоих случаях предполагается, что $\operatorname{det} A \neq 0, \operatorname{det} m \neq 0$. Согласно утверждению 1 любое решение уравнения (1) удовлетворяет (3). Исследуем вопрос о возможности использования формул (15) и (20) в качестве решений исходного уравнения (1).

ЗАмЕчаниЕ 1. Формула (15) единственньм образом определяет решение уравнения (3), тогда как формула (20) зависит от двух постоянных векторов $P^{0}$ и $P^{1}$, выбор которых произволен и приводит к решению $q(x)$ уравнения $(3)$, удовлетворяющему условиям (19). Аналогичный результат имеет место в [1], [2].

УТВЕРЖДЕНИЕ 3. В случае $\operatorname{det} K_{a}(\lambda) \neq 0, \lambda \in(-\infty, \infty)$, решение $q(x) \in H_{1 / 2}^{*}[-a, a]$ уравнения (3) при $f(x) \in H_{1 / 2}^{*}[-a, a]$ удовлетворяет уравнению (1). В случае $\operatorname{det} K_{b}(\lambda) \neq 0, \quad \lambda \in(-\infty, \infty)$, решение $q(x) \in H^{*}[-a, a]$ уравнения (3) при $f(x) \in$ $H^{*}[-a, a]$ удовлетворяет уравнению (1) при условии, что

$$
A P^{0}+B P^{1}=\int_{-a}^{a} f(x) d x, \quad \operatorname{det} B \neq 0 .
$$

Утверждение 3 определяет возможность использования формул (15) и (20) в качестве решения исходного уравнения (1), при этом константы $P^{0}, P^{1}$ в $(20)$ оказываются взаимосвязанными.

6. Пример. Рассмотрим уравнение (1) в виде, которьй встречается, например, в задачах теории упругости [6], [7]. Пусть $N=2$, а компоненты $A_{i j}, B_{i j}$ матриц $A, B$ заданы следуюшим образом: $A_{i j}=B_{i j}=0$, кроме $A_{11}=A_{1}, A_{22}=A_{2}, B_{12}=B_{1}$, $B_{21}=B_{2}$, где $A_{i}, B_{i}$ - некоторые постоянные. В этом случае для компонент матриц $n$, $m$ имеют место выражения: $n_{i j}=m_{i j}=0$, кроме $n_{11}=-B_{1} B_{2} / A_{2}, n_{22}=-B_{1} B_{2} / A_{1}$, 
$m_{11}=m_{1} \equiv A_{1}-\pi^{2} n_{1}, m_{22}=m_{2} \equiv A_{2}-\pi^{2} n_{2}$. Для обеспечения условий $\operatorname{det} A \neq 0$ и $\operatorname{det} m \neq 0$, а также последнего условия (22) положим $A_{k} \neq 0, m_{k} \neq 0, B_{k} \neq 0$.

$\Phi$ ункции $h_{a, b}(s), \Gamma_{0,1}(s)$ в рассматриваемом случае имеют вид

$$
\begin{gathered}
h_{a, b}(s)=\frac{(2 / \pi)^{1 / 2} Z_{a, b}(s)}{\omega_{a, b} \sqrt{1 \pm \omega_{a, b}^{2}}} m^{-1}, \quad \Gamma_{0}(s)=\frac{\pi a \operatorname{ch} \Omega_{b} s}{\sqrt{1-\omega_{b}^{2}} \operatorname{ch} s} n m^{-1}, \\
\Gamma_{1}(s)=\frac{a \operatorname{sh} \Omega_{b} s}{\omega_{b} \operatorname{ch} s} n m^{-1}
\end{gathered}
$$

где $m^{-1}$ - обратная к $m$ матрища, $\omega_{a}^{2}=A_{1} / m_{1}-1 \equiv A_{2} / m_{2}-1, \omega_{b}^{2}=A_{1} / m_{1} \equiv A_{2} / m_{2}$, $\Omega_{a}=\frac{2}{\pi} \operatorname{arcsh} \omega_{a}, \Omega_{b}=\frac{2}{\pi} \arcsin \omega_{b}, Z_{a}(s)=\sin \Omega_{a} s / \operatorname{sh} s, Z_{b}(s)=\operatorname{sh} \Omega_{b} s / \operatorname{sh} s$, при этом условия $\operatorname{det} K_{a}(\lambda) \neq 0, \operatorname{det} K_{b}(\lambda) \neq 0, \lambda \in(-\infty, \infty)$, обеспечиваются, соответственно, неравенствами $0<A_{1} / m_{1} \equiv A_{2} / m_{2}, 0<1-A_{1} / m_{1} \equiv 1-A_{2} / m_{2}$, которые определяют области применимости формул (15) и (20) в качестве решения уравнения (1). Отметим, что функции $h_{a, b}(s)$ и $\Gamma_{0,1}(s)$ вида $(23)$ удовлетворяют условиям (16), (21).

\section{СПИСОК ЦИТИРОВАННОЙ ЛИТЕРАТУРЫ}

[1] Мусхелишвили Н. И. Сингулярные интегральные уравнения. Граничные задачи теории функций и некоторые их приложения к математической физике. М.: Наука, 1968.

[2] Векуа Н. П. Системы сингулярных интегральных уравнений и некоторые граничные задачи. М.: Наука, 1970.

[3] Ильин В.А., Позняк Э. Г. Основы математического анализа. Ч. 1. М.: Наука, 1971; Ч. 2. М.: Наука, 1973.

[4] Беклемишев Д. В. Курс аналитической геометрии и линейной алгебры. М.: Наука, 1974.

[5] Манжиров А. В., Полянин А. Д. Методы решения интегральных уравнений. Справочник. М.: Факториал, 1999.

[6] Галин Л.А. Контактные задачи теории упругости и вязкоупругости. М.: Наука, 1980.

[7] Джонсон К. Механика контактного взаимодействия. М.: Мир, 1989.

Институт проблем механики РАН, г. Москва

Поступило

19.12.2001

Исправленный вариант

14.01.2002 